\title{
Core Foundations that Support the Mobile App Ecosystems
}

\author{
Antoanne Pontes ${ }^{1,2^{*}}$, Mônica Ferreira da Silva ${ }^{1}$, Antonio Juarez Alencar ${ }^{1}$, Eber Assis Schmitz ${ }^{1}$ \\ 1 Tércio Pacitti Institute, Federal University of Rio de Janeiro (UFRJ), Brazil. \\ ${ }^{2}$ Center for Studies and Advanced Systems of Recife (CESAR), Brazil. \\ * Corresponding author. Tel: +55(21)99681-5959; email: antoanne@ufrj.br \\ Manuscript submitted March 19, 2017; accepted May 1, 2017. \\ doi: 10.17706/jsw.12.7.581-597
}

\begin{abstract}
Business transformation through Information Technology has been widely discussed. Nowadays, thanks to the growing number of smartphones, these changes are frequent, and knowledge becomes increasingly more valuable, and to that end, a whole new economic approach take place. The present paper aims to identify new research opportunities motivated by the growth of mobile solutions. It implements a Systematic Literature Review (SLR) in order to understand which are the Core Foundations of the Mobile App Ecosystem, pointing out the current situation of this research area and further strengthening the debate on the most relevant views of this ecosystem. This SLR includes primary studies listed from 2011 through 2016, resulting in a total of 1052 candidate publications, among which 30 publications were selected after a screening process. This paper concludes that academic community research is focused on four topics found within the selected papers, that should describe the Core Foundations of Mobile App Ecosystem: User, Developer, Store, and Publicity. This paper also shows a relationship graph between the selected publications, illustrating the representativeness of these works in this area. It also points out the locations where the Mobile App Ecosystem researches are being conducted. Furthermore, this SLR shows that there is a clear lack of works that combine these four core foundations.
\end{abstract}

Keywords: App economy, app store, mobile platform, software ecosystem.

\section{Introduction}

This research aims to describe a Systematic Literature Review (SLR) looking for the Core Foundations of Mobile App Ecosystem, within formal Computer Science publications. Two main techniques are applied in order to achieve this goal:

- SLR protocol proposed by Kitchenham et al [1]: a means of evaluating and interpreting all relevant researches available on a particular research question, topic area or phenomenon of interest. This protocol uses the Evidence-Based Software Engineering (EBSE) method for aggregating evidence.

- Content analysis method proposed by Bardin [2]: a bottom-up processing method used to discover categories within a text.

The objective of this SLR is to look for research opportunities in Mobile App Ecosystem area. It begins pointing out the relevance of the research topic and the literature background. Followed by Section two, that explains the adopted protocol and how the review process and protocol was conducted, among other points, presenting the research questions and inclusion/exclusion criteria. Section three explains the data extraction process. Section four summarizes the results, presenting the answers to the questions of this research. Section five enumerates some related works and the last Section concludes by presenting data analysis and 
research directions.

\subsection{Relevance}

We are living the information age ${ }^{1}$, according to Davis and Meyer [3]. It represents a new important phase after the Industrial Revolution. From their point of view, changes are constant and knowledge becomes increasingly valuable. Nevertheless, the society has adapted very well to make better use of this new phase, naturally resulting in a new economic approach. New reflections on the social evolution of humanity have been conducted by Brynjolfsson and McAfee [4].

The impact of information technology (IT) on business performance has been widely discussed over the past decade by researchers. Some reports are measuring requirements and benefits of the strategic alignment between business and information technology, as indicated by Reich and Benbasat [5] and Sabherwal and Chan [6]. In this scenario, new business opportunities are emerging, as established by Niederman, Brancheau and Wetherbe [7] and Porter [8], that reinforces the words of Schmidt and Cohen [9]: "Being able to do more in the virtual world will make the mechanics of our physical world more efficient".

Connected machines are now facing a revolution, providing information to improve experiences to customers [10], and the mobile solution is growing up, resulting in competitive advantages provided by technological development. As a matter of fact, we can see greater representation of the mobile app market, as presented in Penny Stocks Lab [11]. Their report shows that smartphone's sales have grown about ten times in the last five years, meaning that there is one smartphone for every 4.5 people on the planet.

Thus, bringing this definition into line with these reports, this research aims to identify the core foundations in which the "mobile app ecosystem" are evolving, in order to investigate possible open issues and research directions.

\subsection{Background}

In 1935, the English botanist and pioneer in the science of ecology, Tansley [12], introduced the concept of an ecosystem when discussed the use of the term biome to define the complex set of creatures who inhabit a specific region.

"Though the organisms may claim for our prime interest, when we are trying to think fundamentally, we cannot separate them from their special environments, with which they form one physical system." Tansley [12].

Contrary to most studies from that time, Tansley brought greater complexity into his area. Studies at that point were limited to systems with organic components only. He recommended that all ecosystem components should be considered, both organic and inorganic. Tansley described an ecosystem as a community of organisms living in harmony with the nonliving components of their environment, such as air, water and mineral soil, interacting as a complex system.

In accordance with Bosch [13], a "software ecosystem" consists of the set of software solutions used in order to enable, support and automate user activities in a social or business environment.

Messerschmitt and Szyperski [14] made use of the words "software" and "ecosystem" together, exemplifying the standard office applications suite, which was found on desktop computers and served to address most user needs. Usually those applications were distributed by the same producer, but we have to take into account that most of them included modules and contents purchased from other producers. Economic enemies are now becoming complementary partners, one of the most important concepts in this new economy, as identified by Hax and Wilde II [15].

According to Wigmore [16], a mobile app is a software application developed specifically for use on small

${ }^{1}$ Also known as the Computer Age, Digital Age, or New Media Age. The period in human history characterized by the shift from traditional industry to an economy based on information computerization. 
wireless computing devices, such as smartphones and tablets, rather than desktop or laptop computers.

This research aims to investigate the evolution of the mobile app ecosystem, a phenomenon that was started as a software ecosystem, evolved into a mobile software ecosystem and currently seems to be evolving to mobile app ecosystem.

\section{Research Protocol}

The adopted method for this SLR follows specialized literature dedicated to Computer Science and was conducted considering an EBSE research practice, according to Kitchenham et al. [1].

Section 2.1 presents a preliminary non-systematic review of the literature. This preliminary review was made in order to identify studies to define and to validate the scope of this research. Section 2.2 shows the research questions used to guide the objective of this SLR. Section 2.3 enumerates the selected electronic sources where the studies were extracted. Section 2.4 defines the search string applied to select the studies. To effectively identify the relevant studies, inclusion and exclusion criteria were inherited from the proposed protocol, as shown in Section 2.5. Ultimately, Section 2.6 lists the limitations and drawbacks which may represent possible bias on the search process.

\subsection{Preliminary Review}

A non-systematic search on relevant literature was used to define the protocol of this research. Websites of major journals in this research area were visited and qualified people were informally consulted, looking for works to guide this research protocol. The works found ([17], [17], [19] and [20]) were used to better direct the search scope and to make the vocabulary more consistent, in order to reach better answers to the Research Questions (RQ).

\subsection{Research Questions}

The research questions (RQ) addressed are:

$\mathrm{RQ1}$. What are the main research topics in the mobile app ecosystem area?

$\mathrm{RQ2}$. Which are the most representative studies on the subject?

$\mathrm{RQ3}$. What are the institutions and authors that lead mobile ecosystem research?

RQ1 intends to identify topics pointed out by researchers in selected papers, based on the categorical analysis method proposed by Bardin [2]. Following to the method, a graded assessment was conducted according to the relevance and frequency of topics identified within each work. Therewith, subjects addressed in each work were identified and grouped into topics according to bottom-up process, an approach where a progression occurs from the individual elements to the whole. The selected topics referred to this question will be presented in Section 4.1.

RQ2 aims to identify papers with higher representation in this subject. Section 4.2 presents a graph with a relation of papers on this SLR subject, through the relationship between references. Besides that, a list of commonly used keywords is presented, using lexical analysis, as suggested by Bardin [2].

RQ3 intends to identify the location and relevance of the institutions and authors that are researching the Mobile App Ecosystem subject, as well the participation of the continents in this research subject. These results will be presented in Section 4.3.

\subsection{Electronic Sources}

This research uses relevant electronic sources dedicated to index Computer Science publications. They are: DBLP Computer Science Bibliography[21], Science Direct [22], CAPES/MEC Portal [23], Google Scholar [24] and Mendeley [25], using each of their available search engines. Approximately 150 million publications were indexed by these electronic sources. 
The electronic source CAPES/MEC Portal was chosen because it is the most representative and widespread portal of periodicals among researchers in Brazil. It is a database that gathers international scientific production and currently has a collection of more than 38,000 full-text periodicals.

Mendeley was selected as another electronic source, and not just as a software to manage data from selected publications. The function available in the desktop version which recommends similar publications was used. According to Knight [26], "the recommendations come from a mix of analysis based on the content of the selected paper and what other users with similar interests are reading".

\subsection{Search String}

In order to answer the research questions (RQ), the most relevant keywords have been chosen to compose an efficient search string. A lot of combinations were tried in order to return the works found in the preliminary review. The chosen search string that brought more relevant contributions was "mobile AND ecosystem".

This search string, in turn, was adapted to be used in each electronic source, respecting their technical requirements to search in the titles and abstracts of publications available in each of them. This process was carried out individually into each electronic source. All extracted data were imported to Mendeley, generating one record for each publication found. The list of formatted strings applied in each electronic source can be found in Table 1.

Table 1. Formatted Strings for Each Electronic Source

\begin{tabular}{l|l}
\hline \hline Electronic Source & \multicolumn{1}{c}{ Formatted String } \\
\hline DBLP & mobile ecosystem \\
Science Direct & mobile and ecosystem[All Sources(Computer Science)] \\
CAPES/MEC Portal & mobile AND ecosystem \\
Google Scholar & allintitle: mobile (ecosystem/ecosystems) \\
Mendeley & search by similar papers. \\
\hline \hline
\end{tabular}

Especially for the search in CAPES/MEC Portal, the string was used to make a direct search in the titles and abstracts of available publications, and received an additional filter composed of other required keywords, to exclude publications that do not belong to the literature of computer science, e.g. Ecology Studies, Sustainable Development, Habitat, Marine, Ecology, Biodiversity, Conservation, Fish, Predators, Landscape, Forests and Sediments.

For Google Scholar, the search string was applied only in the titles of publications. In contrast, applying the search string in the whole content of the papers makes it impractical to use the result, due to the huge volume of items out of context.

As previously pointed, Mendeley was used with "similar items" functionality, available in the software, to get suggestions of some other similar papers based on researchers' database.

\subsection{Inclusion and Exclusion Criteria}

Formal papers published in Computer Science area were included in this research. These papers, which should contain information about the mobile app market, users, and the ecosystem, were initially filtered by the inclusion/exclusion criteria as proposed by Petersen et al. [27], and can be found in Table 2.

Publications available before 2011 were not used in this SLR. This was purposely done in order to identify the most representative contributions of the largest growth momentum in the mobile market [11]. 
Table 2. Inclusion and exclusion criteria.

\begin{tabular}{|c|c|}
\hline Inclusion criteria & Exclusion criteria \\
\hline $\begin{array}{l}\text { Publications available since 2011; } \\
\text { Formal publications; } \\
\text { Preliminary analysis, reviews or trends about mobile } \\
\text { app ecosystem; } \\
\text { Papers that describe the main research topics in } \\
\text { mobile app ecosystem. }\end{array}$ & $\begin{array}{l}\text { Papers in areas other than Computer Science, such as agriculture, } \\
\text { medicine, etc.; } \\
\text { Informal publications; } \\
\text { Papers that do not present enough empirical evidence; } \\
\text { Working Papers (incomplete papers or papers under development); } \\
\text { Papers not written in English; } \\
\text { Papers that address mobile network physical environments, or that } \\
\text { address platform features, such as payment systems; } \\
\text { Papers that approach a specific application. }\end{array}$ \\
\hline
\end{tabular}

\subsection{Limitations and Drawbacks}

This SLR was structured in accordance with the protocol proposed by Kitchenham et al. [1]. Moreover, it was understood that there may be possible limitations and drawbacks to be considered during validity execution in order to address bias and internal and external validity. E.g. it is possible that some studies were not chosen according to this defined search process, representing possible bias on searching process.

Sepúlveda, Cravero and Cachero[28] pointed out that 88\% of SLR whose papers did not consider the possible threats to validity. Trying to mitigate possible bias in excluding relevant papers, a non-systematic search was used as the basis for defining the adopted protocol, as pointed out in Section 2.1. Inclusion/exclusion criteria were manually applied by authors, sometimes there were some difficulties to detect the relevant information of certain items, which limited the process of information extraction from the selected papers. For example, some of the papers did not focus explicitly on the main subject, and the search was limited to some relevant electronic sources.

\section{Data Extraction Process}

This Section describes the data extraction process steps and quantitatively summarizes the results of this research. The process was performed in the fourth week of December 2016 to ensure that all this year's publications, or the largest amount of them, were included.

\subsection{Search Process Steps}

The applied search resulted initially in 1052 items, of which 940 items remained after removing duplicated records. Then the inclusion/exclusion criteria were applied to titles and abstracts resulting in 56 items. At this point, a different technique was applied. A Mendeley function was used to select and include more 92 similar items. Total items were also filtered through the inclusion and exclusion criteria, resulting in 81 relevant items. Lastly, the abstracts and full-text articles of these relevant items were read. From this amount, 30 publications were finally selected. Figure 1 shows the SLR process with its steps in sequence and a summary of the number of publications in each step.

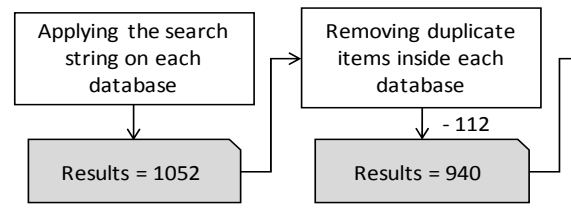

\subsection{Publications Selection}

Following the suggestion of Kitchenham et al. [1], Table 3 shows all publications found in each electronic source, grouped by year. "Found" represents the number of publications before execution of the inclusion and exclusion criteria process. "Relevant" shows a number of items after execution of inclusion and exclusion 
criteria process. Finally, we have the list of "Selected" items, which stands for the number of publications after full text reading process.

Table 3. Results of Each Electronic Source by Year.

\begin{tabular}{r|ccccccc||c}
\hline \hline Electronic source \Year & 2011 & 2012 & 2013 & 2014 & 2015 & 2016 & 2017 & Total \\
\hline DBLP Found & 16 & 8 & 12 & 11 & 27 & 16 & 1 & 91 \\
Relevant & 3 & 5 & 5 & 6 & 9 & 4 & 0 & 32 \\
Selected & 2 & 1 & 2 & 0 & 1 & 0 & 0 & 6 \\
Science Direct & & & & & & & & \\
Found & 3 & 6 & 7 & 6 & 17 & 11 & 3 & 53 \\
Relevant & 1 & 1 & 0 & 3 & 1 & 2 & 1 & 9 \\
Selected & 0 & 0 & 0 & 1 & 1 & 2 & 0 & 4 \\
CAPES/MEC Portal & & & & & & & & \\
Found & 61 & 75 & 133 & 132 & 119 & 159 & 11 & 690 \\
Relevant & 6 & 6 & 6 & 10 & 7 & 8 & 0 & 43 \\
Selected & 3 & 1 & 0 & 3 & 4 & 3 & 0 & 14 \\
Google Scholar & & & & & & & & \\
Found & 38 & 29 & 39 & 40 & 41 & 29 & 2 & 218 \\
Relevant & 4 & 8 & 7 & 4 & 8 & 6 & 0 & 37 \\
Selected & 2 & 3 & 4 & 1 & 3 & 2 & 0 & 15 \\
Fendeley & & & & & & & & \\
Found & 25 & 14 & 29 & 14 & 8 & 3 & 0 & 93 \\
Relevant & 5 & 3 & 5 & 7 & 1 & 3 & 0 & 24 \\
Selected & 1 & 1 & 3 & 3 & 0 & 2 & 0 & 10 \\
\hline \hline
\end{tabular}

\subsection{Trend line Representation}

It is possible to notice a representative increase in the number of papers as pointed by a linear trend line ${ }^{2}$, as shown in Figure 2. This Figure illustrates the year-wise distribution of selected papers using a trend line representation. This increase may reflect the growth of the mobile market, as indicated by Penny Stocks Lab reports [11].

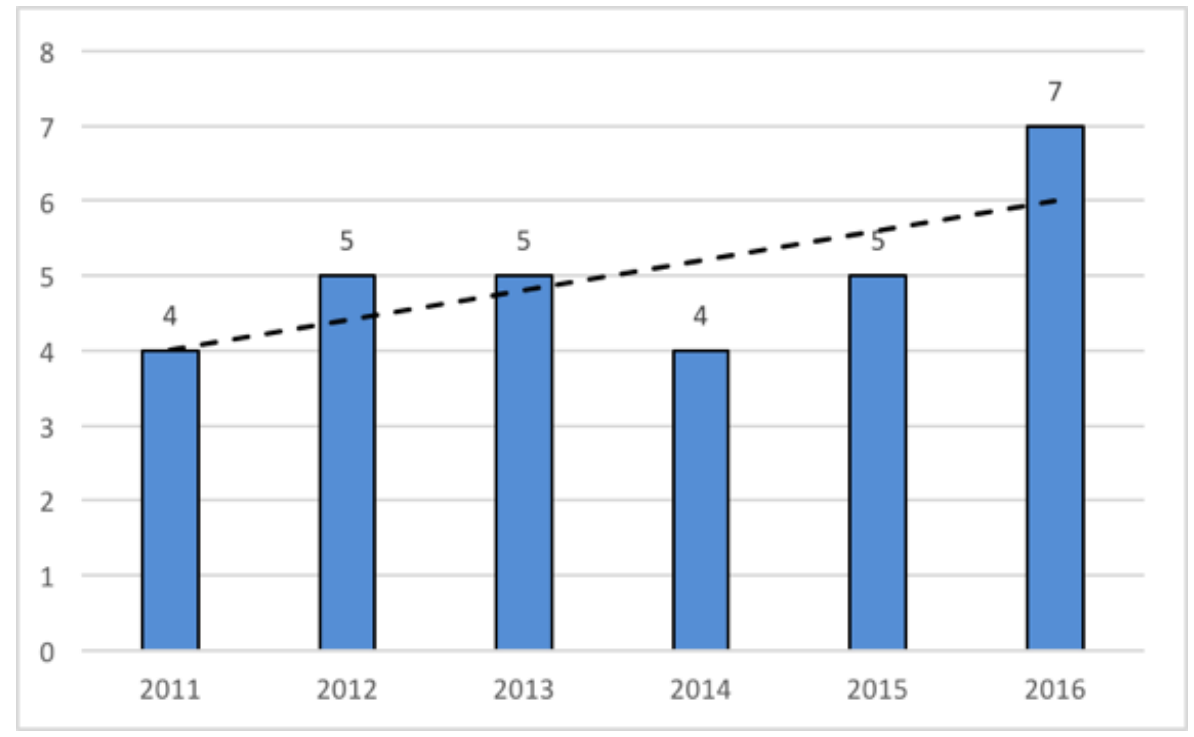

Fig. 2. Year-wise distribution with a linear trend line.

\subsection{Electronic Sources Representativeness}

The representativeness of each electronic source on this subject, in the number of available publications 
and selected items, is displayed in the Figure 3. CAPES/MEC Portal [23] and Google Scholar [24] are similar in relation to the number of selected items. A good representativeness of the Mendeley database of similar items is also perceived. It seems that this mechanism of suggesting similar publications is a valid one.

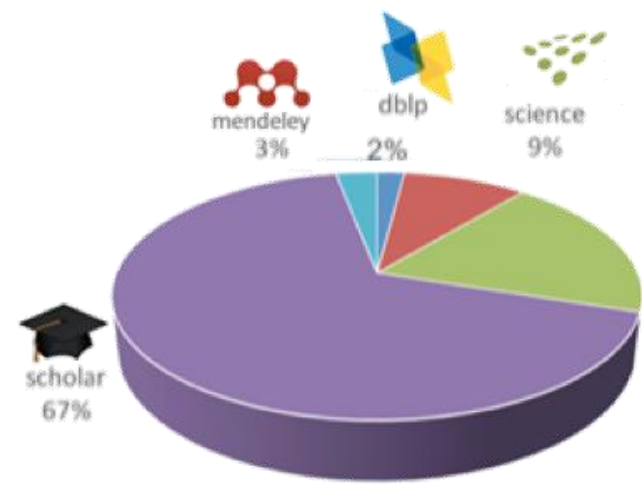

Available items

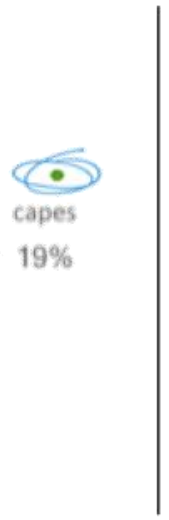

Fig. 3. Electronic sources and number of selected items.

\subsection{Electronic Sources Intersection}

To better understand the relationship between each electronic source, Table 4 shows the intersection of publications among the 30 selected ones.

Table 4. Number of Intersection of Selected Papers from each Electronic Source

\begin{tabular}{l|ccccc}
\hline \hline \multicolumn{1}{c|}{ Intersection } & DBLP & Science Direct & CAPES/MEC Portal & Google Scholar & Mendeley \\
\hline DBLP & 8 & & & & \\
Science Direct & 1 & 5 & 12 & & \\
CAPES/MEC Portal & 0 & 3 & 0 & 13 & \\
Google Scholar & 8 & 1 & 1 & 3 & 8 \\
Mendeley & 2 & 0 & \multicolumn{2}{c}{ Unique selected papers: 30} \\
\hline \hline
\end{tabular}

\section{Search Results}

This Section summarizes the results of this SLR, in order to answer each RQ presented in Section 2.2. A unique index (Research Id) identifies the 30 selected papers, and is referenced in all tables. The complete list of selected papers can be found in Table 10 of Appendix Section.

\subsection{Publications Content}

\subsubsection{Main discussed subjects}

As previously mentioned in Section 2.2, the classification criteria follow the method proposed by Bardin [2]. The author emphasizes the possibility of splitting elements, starting from particular items and reassembling them gradually so as to assign a category title. According to Bardin, content analysis means to say no "to the simple reading of the real", and to accept the provisional nature of the hypothesis, experiments or research plans.

Answering RQ 1, Table 5 reports each paper and its subject, showing what is being mainly addressed as research subjects. 
Table 5. Main Discussed Subjects

\begin{tabular}{l|l}
\hline \multicolumn{1}{c|}{ Subject } & \multicolumn{1}{|c}{ Research ID } \\
\hline User & $1,3,8,11,12,14,19,20,22,23,24,25,26,27,28,29,30$. \\
Developer & $2,3,4,7,8,10,14,16,17,18,24,26,29,30$. \\
Store & $1,2,3,7,8,9,13,15,18,21,22,25$. \\
Publicity & $5,6,10,11,26,28$. \\
\hline \hline
\end{tabular}

From the selected publications, the most common related subject is "User", with 17 occurrences. These publications present how the application can be found by users at the store navigation list, intention of use, acceptance of an app, and how it is focused on the user's experience (UX). On newer papers, the subject "User" has also a good representativeness, as pointed out in Table 5 through underline marking.

Following the first findings, another common subject is "Developer", placed second, with 14 occurrences. It describes the performance when a platform is being actively used, under a developer perspective. This relates to financial return, one that directly represents the number of app downloads and usability within the development environment, which can be a decisive factor on platform adoption.

Third, but not far from the second, is the subject "Store", with 12 occurrences, representing marketplaces and references about its business model and strategy. This question mainly appears in the form of a comparison between the two most important mobile application marketplaces, the Apple AppStore and Google Play, but it can be noticed that they are all essentially similar. The main difference is about business models, app marketplace platform, and ways to connect app developers and app users, no matter how open or closed it is.

Ultimately, with 6 occurrences, "Publicity" is another main subject. It focus on dissemination and marketing strategies. This subject is directly related to the marketing strategy implemented by the app publisher or producer. It means that, the more is invested in advertising campaigns, the greater the chances of an app being found by the user among thousands of other apps.

Figure 4 shows the summary of the subjects found in the selected papers, that can represent the Core Foundations of Mobile App Ecosystem. It can be perceived a strong tendency of studies focused on the acquisition of user experience applications, since the most representative subject is "User", followed closely by "Developer" and "Store", with a very little difference.

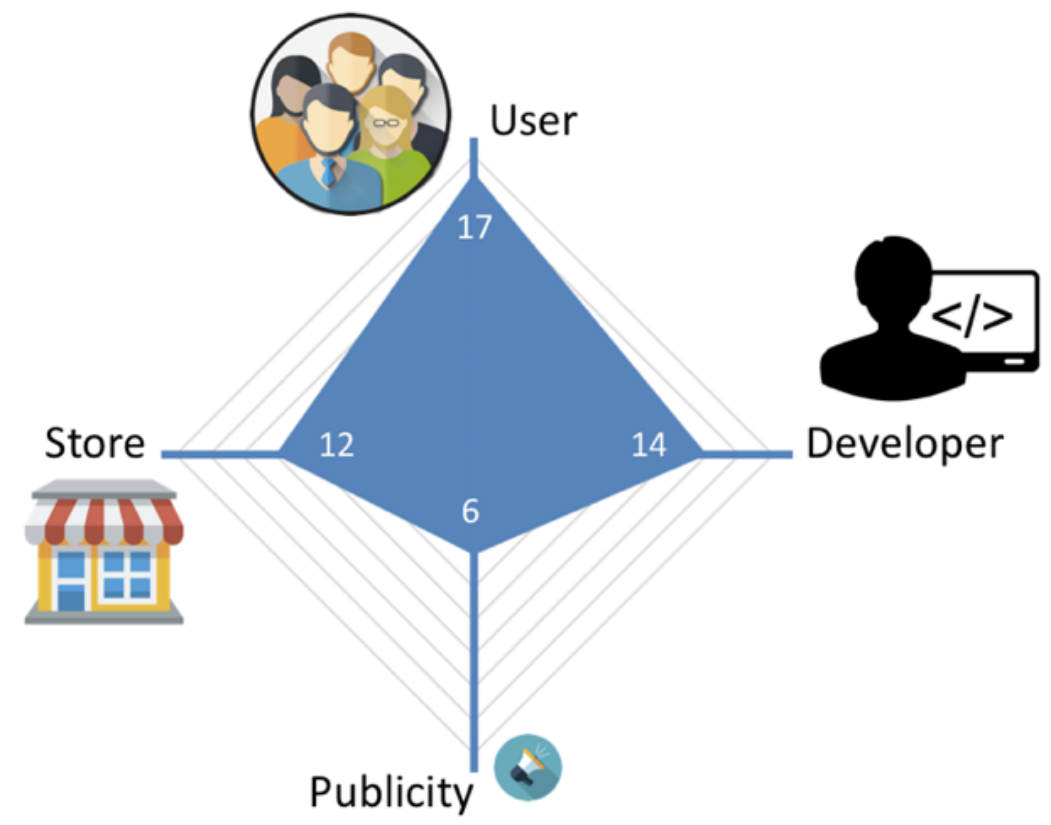

Fig. 4. The main discussed subjects in mobile app ecosystem. 


\subsubsection{Trending Topics}

Figure 5 shows a word cloud extracted through paper abstracts, as proposed by Sepúlveda, Cravero and Cachero[28]. Through this word cloud, can be extracted a first impression of the subjects addressed by the 30 selected papers.

It is notable that there are similar words, such as "developer" and "developers", because the abstracts were not processed, understanding that words in the singular or plural can represent different contexts. Note that the word cloud trending topics are aligned with this SLR main subject, thus supporting the selection of these papers. It was created using Wordle 3 , a web tool for generating "word clouds" from provided text.

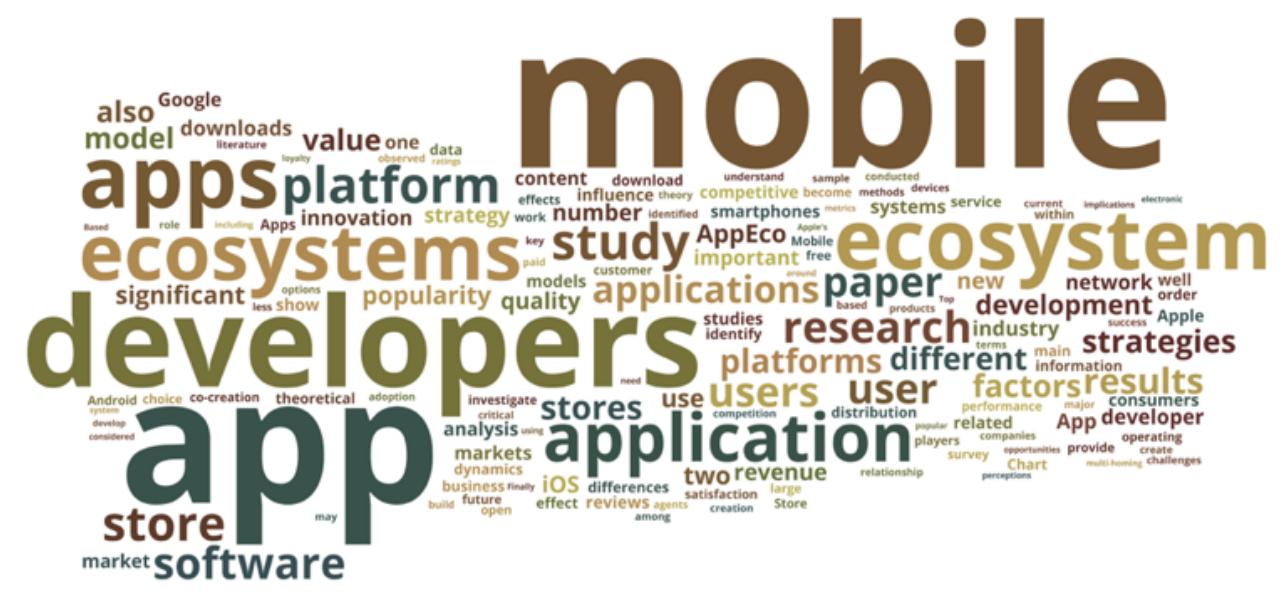

Fig. 5. Word cloud from the abstracts of selected papers.

\subsubsection{Keywords List}

The keywords list, shown in Table 6, presents the most common referenced terms under selected papers. The most referenced keywords from the selected papers were used for qualify this research. They are: "Mobile Platform" and "App Store", followed by "Software Ecosystem" and "App Economy". It reinforces the relationship of the search string with the result of this SLR through the selected papers with the main subject of this research, supporting the selection of papers during the preliminary review, as shown in Section 2.1.

Table 6. General List of Keywords from Selected Papers

\begin{tabular}{l|c}
\hline \multicolumn{1}{c|}{ Keyword } & Qty \\
\hline Mobile Platform & 8 \\
App Store & 7 \\
Software Ecosystem & 5 \\
App Economy & 4 \\
Agent-based Simulation & $\mathbf{3}$ \\
App Popularity & $\mathbf{3}$ \\
Business Model & $\mathbf{3}$ \\
Mobile Apps & $\mathbf{3}$ \\
\hline \hline
\end{tabular}

\subsection{Relevance of Studies}

\subsubsection{Cross reference}

A relationship graph between the 30 selected papers can be found in the Figure 6, pointing out which are self-references papers and how they are arranged. This graph shows that the paper ID 30 (marked in green color) implements more references from selected papers by this SLR. It may mean that this paper can be the nearest to the subject addressed in this SLR - i.e., Mobile App Ecosystem.

3 http://www.wordle.net 
The paper with ID 3 (marked in navy color) can be considered as the most referenced (by 6 papers: 9, 10, $16,18,22$, and 30 ), which can mean high representation in this subject. In contrast, the papers that are not referenced and not being referenced within this group can be called "outliers" papers, they may be points of connection with other subjects, e.g. publications 4, 8, 12,16, and 19.

It can also be seen that an interesting sequence marked in blue color (by papers $3,9,18,21$, and 26), that point to the evolution of a research topic, originated by the publication of ID 3.

Another interesting model is the representation of "grouped" papers, as we can perceive in the graph by publications $5,6,11,20$, and 29 . It may be representing a proximity of the addressed issues, in this case, these papers were generated by the same research group.

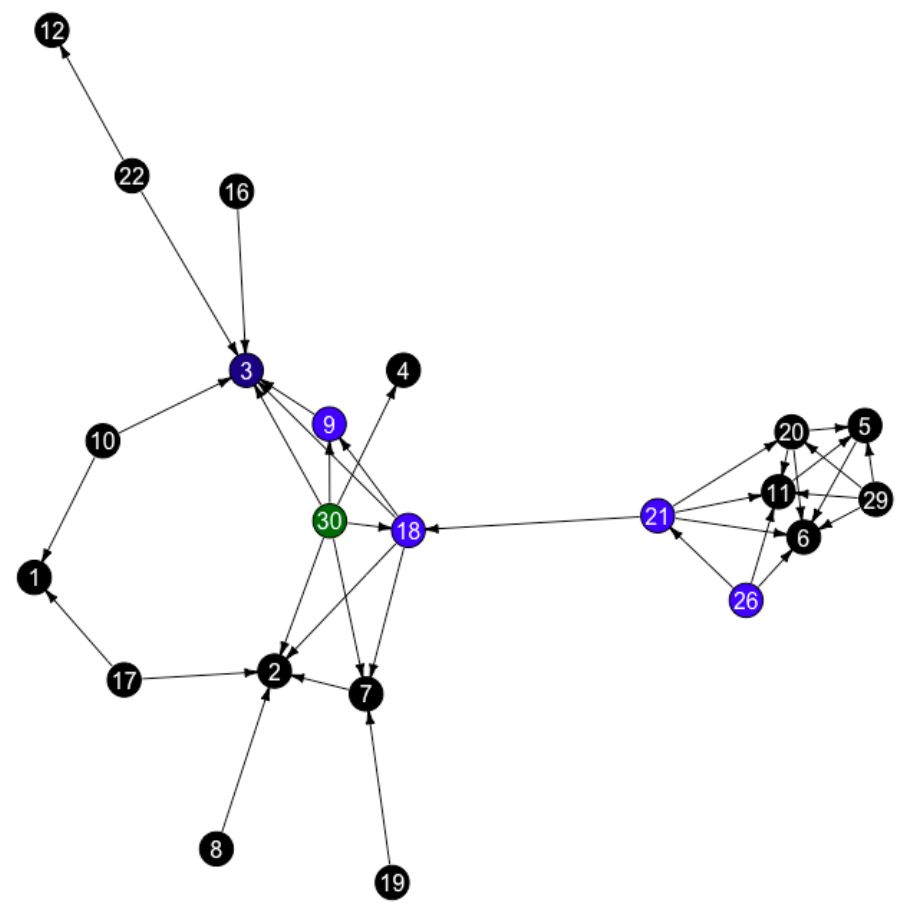

Fig. 6. Graph representing the relationship between selected papers.

\subsubsection{List of Venues}

Another way to represent the relevance of selected papers is the list of venues, that recognizes and comprehends the journals and conferences that have been addressed in this research. They were published through the journals list, as shown in Table 7, and by the conferences list, as shown in Table 8.

Table 7. List of Journals

\begin{tabular}{l} 
Name of Journal \\
\hline ACM SIGEVOlution \\
Business \& Information Systems Engineering \\
Computers in Human Behavior \\
IEEE Communications Magazine \\
IEEE Transactions on Evolutionary Computation \\
IEEE Transactions on Software Engineering \\
Information \& Management \\
Information and Software Technology \\
Issues in Information Systems \\
Journal of Management Information Systems \\
Journal of Systems and Software* \\
Journal of Theoretical and Applied Electronic Commerce Research*
\end{tabular}


Service Science*

Telematics and Informatics*

* Journals with more than one publication from selected papers.

Table 8. List of Conferences

\begin{tabular}{l|l}
\hline \hline Acronym & \multicolumn{1}{|c}{ Conference Name } \\
\hline SBQS & Brazilian Symposium on Software Quality \\
COMPSAC & Computer Software and Applications Conference \\
ECRA & Electronic Commerce Research and Applications \\
ECSAW & European Conference on Software Architecture Workshops \\
ITS & European Regional Conference of the International Telecommunication Society \\
HICSS & Hawaii International Conference on System Sciences \\
IEEE CEC & IEEE Congress on Evolutionary Computation \\
ICIS & International Conference on Information Systems \\
ICIN & International Conference on Intelligence in Next Generation Networks \\
ALIFE & International Conference on the Synthesis and Simulation of Living Systems \\
IMC & Internet Measurement Conference \\
\hline \hline
\end{tabular}

\subsection{Leading in Mobile App Ecosystem Subject}

\subsubsection{Institutions by continent}

Table 9 presents the number of researchers by institutions that are contributing to Mobile App Ecosystem subject, grouped by continent. Overall, the set of studies is dominated by European researchers, who have been involved in 19 of the 30 selected studies. In particular, the University College London has been involved in 4 of the studies ([19], [20], [30], and [31]).

Table 9. Number of Authors of Institutions by Continent

\begin{tabular}{l|c}
\hline \hline \multicolumn{1}{c|}{ Continent } & Researchers by Institutions \\
\hline Europe & 19 \\
North America & 9 \\
Asia & 8 \\
South America & 3 \\
Australia & 1 \\
Africa & 0 \\
\hline \hline
\end{tabular}

\subsubsection{Representativeness of Institutions on the Planet}

Figure 7 shows a map with the representativeness of the institutions where the 30 selected papers were written. So, we can identify the regions of the planet that are most focused on contributing to this area.

To elaborate these markings on the map, the institutions of each author of all 30 selected papers were found. LatLong.net ${ }^{4}$ was used to find a correct geolocation of each institution, using the name of a place, city, state, or address to get latitude and longitude coordinates on the map. Then, Google Maps JavaScript API ${ }^{5}$ were used to build a customized map with markings, that represents the number of researchers by institution involved in Mobile App Ecosystem subject.

\subsubsection{Major Contributors}

The researchers who most contributed to the subject were Soo Ling Lim and Peter J. Bentley, both affiliated to University College London. They contributed with 5 papers within this SLR. Apparently, they are the two most active authors that are researching the same subject together. The University of Turku had 6 researchers involved in this research area. They are responsible for 3 of the 30 selected papers.

4 http://www.latlong.net

5 https://developers.google.com/maps/documentation/javascript 
This map marking helps us to understand any relationship between market behavior, e.g. the growth of volume in smartphone sales, with relevant studies in the area.

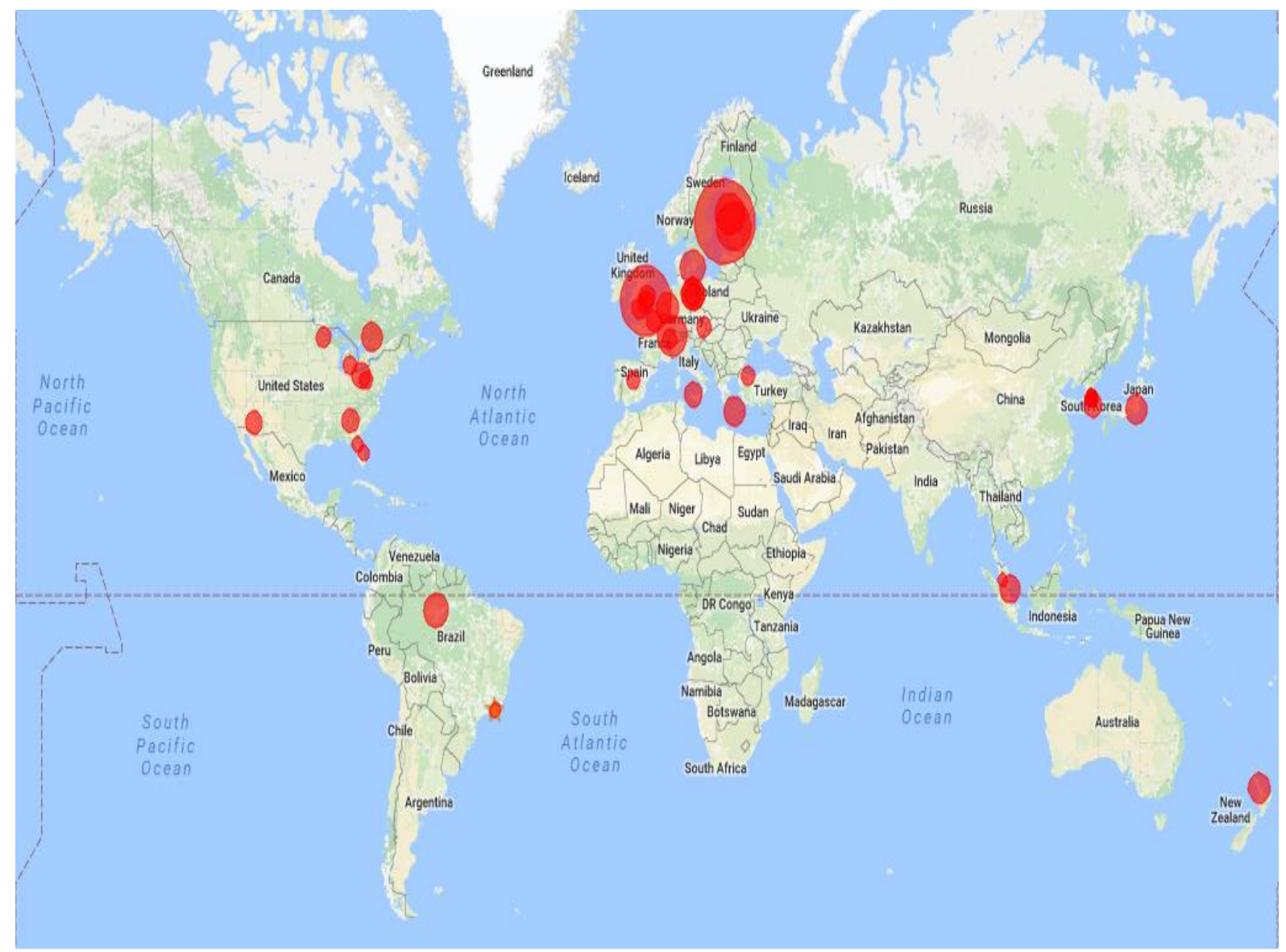

Fig. 7. Institutions placement on the planet.

\section{Related Works}

This Section aims to point out similarities and differences between this research and contributions of related works.

Fontão, Santos and Dias-Neto [17] bring us a vision of Software Ecosystem (SECO) in the mobile platform domain, naming it as Mobile Software Ecosystem (MSECO). They aim to identify characteristics and how this kind of ecosystem is related to the use of the mobile platform by users, with social or business goal. They present a Systematic Mapping protocol, whereas this paper points out a Systematic Literature Review. While the Systematic Mapping intends to map and present the current state of research on a particular subject, a Systematic Literature Review addresses specific questions about a certain subject. According to the authors, Software Ecosystem (SECO) consists of a set of software solutions that enables, supports and automates the activities and transactions by the actors in the associated social or business ecosystem and the organizations that provide these solutions through a software platform. On the other hand, a MSECO is a set of collaborative systems, users and developers that have an emotional link to the platform and/or brand. They create complex relationships paradoxically driven by competition and cooperation within niches. In this sense, a MSECO is similar to a biological ecosystem.

Barbosa and Alves [32] objective is to understand how software ecosystem is being researched. In addition, they try to point out the benefits, challenges and limitations. The authors explore desktop environments, 
unlike this research, which aims to explore mobile environments. The mapping study confirms that software ecosystem is an emergent field, and indicates that software ecosystem research has been mainly inspired by studies from business and natural ecosystems, as well as traditional software engineering research on software product lines, software evolution, and open source software. Their preliminary opinion is that software ecosystems need more studies to increase body of evidence.

The main difference between Manikas and Hansen [33] and this research is that these authors aim to define software ecosystem in a platform-agnostic view, whereas this research address this subject within mobile platform. To ensure that the literature review is systematic and to minimize researcher bias, they used Kitchenham et al. [1] protocol as well as this research. Their literature extraction consisted of two separated keywords: "software" and "ecosystem". Their research, which was conducted with publications between 2007 and 2011, found a total of 212 papers extracted from a list of scientific libraries. From this total, 59 were selected as reporting research relevant to the field of SECO.

The first paper presents a Systematic Mapping on software ecosystems in general, the second one is dedicated to desktop environment, and the last one, besides being out of date, presents a platform-agnostic view. Thus, there is a clear gap of current Systematic Literature Reviews dedicated to Mobile App Ecosystems.

\section{Conclusion}

Mobile App Ecosystems have taken on an increasingly important role in the new economic vision pointed out by Davis and Meyer [3]. The relationship between business and IT strategic objectives are interlaced with the advancement of technology, according to Reich and Benbasat [5].

Contrary to Tansley's [12] approach, none of the authors of the 30 selected papers addressed the four subjects at the same time ("User", "Developer", "Store", and "Publicity") and the relationship between them. Those publications presented each subject in isolation, except for some cases that two or three subjects are covered in the same research, as presented in Table 10.

Among the selected publications, it is noteworthy that none have presented possible external factors that influence the Mobile App Ecosystem.

During this SLR, it is noticeable that just like the four natural elements of the ecosystem in which we live (air, earth, fire, and water), the subjects presented serve as the foundations that sustain and maintain the balance of Mobile App Ecosystem, that is, the core foundations of this type of ecosystem.

It can be seen that mobile market growth is a reality [11]. Nevertheless, we haven't seen this growth in the same rate in published research about Mobile App Ecosystem, as shown in Figure 2.

Figure 6 presents a graph showing the relationship between selected papers, which suggests that paper 3 is the most representative for this set, since it was the most cited. Besides, it is noticeable the interaction of a research group who publish in this subject.

The four common subjects are pointed out by this SLR. Table 5 lists "User" as the most common and recently explored subject related to the four latest published papers, and Figure 4 confirms that it is indeed one of the most representative subjects.

\subsection{Research Directions}

Even answering the Research Questions, there are some other open questions. In this Section, these issues will be listed in order to propose new research directions based on Mobile App Ecosystem.

The core foundations on which this research subject is supported have been identified here. They are "User", "Developer", "Store", and "Publicity". An actual challenge is to better understand how each subject can influence, limit or interact with this ecosystem.

According to the ecologist Tansley [12], an ecosystem is composed of various elements that interact with 
each other. Thus, we cannot understand the growth of a variable in isolation but rather we should look for its influence at the ecosystem as a whole.

It is also important to discover if there are other indirect factors impacting in the Mobile App Ecosystem balance. The occurrence of an event, such as, the Olympic Games or a catastrophe, may influence users to adopt an app.

Improve mobile environment simulators to take these four pillars in consideration, as pointed out from this research, could be another future research direction.

Another topic emerging from this SLR is the need to elaborate a conceptual model that represents the entities of this ecosystem and their relationships. This can be done by using a semantic web based on ontological foundations like the Unified Foundational Ontology (UFO) [34].

Lastly, it might be interesting to investigate why continents such as South America, that have one of the largest smartphone markets, are not among those which are more active in researches on the subject.

\section{Appendix}

Table 10. List of all References of Selected Papers Sorted by Year

\begin{tabular}{|c|c|c|c|c|c|c|c|}
\hline Id & Authors & Title & Year & $\mathrm{U}$ & $\mathrm{D}$ & S & $\mathrm{P}$ \\
\hline 1 & Müller et al. & $\begin{array}{l}\text { A comparison of inter-organizational business models of mobile app stores: } \\
\text { There is more than open vs. closed }\end{array}$ & 2011 & $\mathrm{X}$ & & $\mathrm{X}$ & \\
\hline 2 & Holzer and Ondrus & Mobile application market: A developer's perspective & 2011 & & $\mathrm{X}$ & $\mathrm{X}$ & \\
\hline 3 & Basole and Karla & On the Evolution of Mobile Platform Ecosystem Structure and Strategy & 2011 & $\mathrm{X}$ & $\mathrm{X}$ & $\mathrm{X}$ & \\
\hline 4 & Schultz et al. & $\begin{array}{l}\text { The new role of developers in the mobile ecosystem: An Apple and Google } \\
\text { case study }\end{array}$ & 2011 & & $\mathrm{X}$ & & \\
\hline 5 & Lim and Bentley & $\begin{array}{l}\text { App Epidemics: Modelling the Effects of Publicity in a Mobile App } \\
\text { Ecosystem }\end{array}$ & 2012 & & & & $\mathrm{X}$ \\
\hline 6 & Lim and Bentley & $\begin{array}{l}\text { How to be a Successful App Developer: Lessons from the Simulation of an } \\
\text { App Ecosystem }\end{array}$ & 2012 & & & & $\mathrm{X}$ \\
\hline 7 & $\begin{array}{l}\text { Cuadrado and } \\
\text { Dueñas }\end{array}$ & $\begin{array}{l}\text { Mobile application stores: Success factors, existing approaches, and future } \\
\text { developments }\end{array}$ & 2012 & & $\mathrm{X}$ & $\mathrm{X}$ & \\
\hline 8 & Kajanan et al. & $\begin{array}{l}\text { Takeo and Sustained Success of Apps in Hypercompetitive Mobile Platform } \\
\text { Ecosystems: an Empirical analysis }\end{array}$ & 2012 & $\mathrm{X}$ & $\mathrm{X}$ & $\mathrm{X}$ & \\
\hline 9 & Basole and Karla & $\begin{array}{l}\text { Value Transformation in the Mobile Service Ecosystem: A Study of App } \\
\text { Store Emergence and Growth }\end{array}$ & 2012 & & & $\mathrm{X}$ & \\
\hline 10 & Deniz and Kehoe & $\begin{array}{l}\text { Fostering innovation: Factors that attract and retain third party developers } \\
\text { in mobile ecosystems }\end{array}$ & 2013 & & $\mathrm{X}$ & & $\mathrm{X}$ \\
\hline 11 & Lim and Bentley & $\begin{array}{l}\text { Investigating App Store Ranking Algorithms Using a Simulation of Mobile } \\
\text { App Ecosystems }\end{array}$ & 2013 & $\mathrm{X}$ & & & $\mathrm{X}$ \\
\hline 12 & Petsas et al. & $\begin{array}{l}\text { Rise of the planet of the apps: A Systematic Study of the Mobile App } \\
\text { Ecosystem }\end{array}$ & 2013 & $\mathrm{X}$ & & & \\
\hline 13 & Lee and Kim & Strategy scenario selection in the competition of mobile ecosystems & 2013 & & & $\mathrm{X}$ & \\
\hline 14 & Haenni et al. & $\begin{array}{l}\text { A Quantitative Analysis of Developer Information Needs in Software } \\
\text { Ecosystems }\end{array}$ & 2014 & $\mathrm{X}$ & $\mathrm{X}$ & & \\
\hline 15 & Lee and Raghu & Determinants of Mobile Apps' Success: Evidence from the App Store Market & 2014 & & & $\mathrm{X}$ & \\
\hline 16 & Ryu et al. & Factors affecting application developers' loyalty to mobile platforms & 2014 & & $\mathrm{X}$ & & \\
\hline 17 & $\begin{array}{l}\text { Koch and } \\
\text { Kerschbaum }\end{array}$ & $\begin{array}{l}\text { Joining a smartphone ecosystem: Application developers' motivations and } \\
\text { decision criteria }\end{array}$ & 2014 & & $\mathrm{X}$ & & \\
\hline 18 & Hyrynsalmi et al. & Sources of Value in Application Ecosystems & 2014 & & $\mathrm{X}$ & $\mathrm{X}$ & \\
\hline 19 & Hyrynsalmi et al. & $\begin{array}{l}\text { Busting Myths of Electronic Word of Mouth: The Relationship between } \\
\text { Customer Ratings and the Sales of Mobile Applications }\end{array}$ & 2015 & $\mathrm{X}$ & & & \\
\hline & Lim et al. & $\begin{array}{l}\text { Investigating Country Differences in Mobile App User Behavior and } \\
\text { Challenges for Software Engineering }\end{array}$ & 2015 & $\mathrm{X}$ & & & \\
\hline 21 & $\begin{array}{l}\text { Fontao, Santos and } \\
\text { Dias-Neto }\end{array}$ & Mobile Software Ecosystem (MSECO): A Systematic Mapping Study & 2015 & & & $\mathrm{X}$ & \\
\hline 22 & Lee & Reliability of Mobile App Ecosystem & 2015 & $\mathrm{X}$ & & $X$ & \\
\hline 23 & Kim et al & The effects of quality on the satisfaction and the loyalty of smartphone users & 2015 & $\mathrm{X}$ & & & \\
\hline
\end{tabular}




\begin{tabular}{|c|c|c|c|c|c|c|}
\hline & Liu et al. & $\begin{array}{l}\text { A study of the effects of social factors and innovation characteristics on } \\
\text { search effort and uncertainty in mobile app adoption }\end{array}$ & 2016 & $\mathrm{X}$ & $\mathrm{X}$ & \\
\hline 25 & $\begin{array}{l}\text { Genc-Nayebi and } \\
\text { Abran }\end{array}$ & $\begin{array}{l}\text { A Systematic Literature Review: Opinion Mining Studies from Mobile App } \\
\text { Store User Reviews }\end{array}$ & 2016 & $\mathrm{X}$ & & $\mathrm{X}$ \\
\hline 26 & Gomes et al & $\begin{array}{l}\text { An Empirical Analysis of Mobile Apps' Popularity Metrics in Mobile } \\
\text { Software Ecosystems }\end{array}$ & 2016 & $\mathrm{X}$ & $\mathrm{X}$ & $\mathrm{X}$ \\
\hline 27 & Wong et al. & Mobile environments and innovation co-creation processes \& ecosystems & 2016 & $\mathrm{X}$ & & \\
\hline 28 & $\begin{array}{l}\text { Roma and } \\
\text { Ragaglia }\end{array}$ & $\begin{array}{l}\text { Revenue models, in-app purchase, and the app performance: Evidence from } \\
\text { Apple's App Store and Google Play }\end{array}$ & 2016 & $\mathrm{X}$ & & $\mathrm{X}$ \\
\hline 29 & Lim et al & $\begin{array}{l}\text { The Effects of Developer Dynamics on Fitness in an Evolutionary Ecosystem } \\
\text { Model of the App Store }\end{array}$ & 2016 & $\mathrm{X}$ & $\mathrm{X}$ & \\
\hline 30 & Hyrynsalmi et al. & $\begin{array}{l}\text { The influence of developer multi-homing on competition between software } \\
\text { ecosystems }\end{array}$ & 2016 & $\mathrm{X}$ & $\mathrm{X}$ & \\
\hline
\end{tabular}

\section{References}

[1] Kitchenham, B., Brereton, O. P., Budgen, D., Turner, M., Bailey, J., \& Linkman, S. (2009). Systematic literature reviews in software engineering. A Systematic Literature Review. Information and Software Technology.

[2] Bardin, L. (1977). Content analysis. Editions Lisbon.

[3] Davis, S., \& Meyer, C. (1999). Blur: The speed of change in the connected economy. Ernst \& Young Center for Business Innovation - Capstone.

[4] Brynjolfsson, E., \& McAfee, A. (2014). The second machine age: work, progress, and prosperity in a time of brilliant technologies. WW Norton \& Company.

[5] Reich, B. H., \& Benbasat, I. (1996). Measuring the linkage between business and information technology objectives. MIS quarterly.

[6] Sabherwal, R., \& Chan, Y. E. (2001). Alignment between business and IS strategies: A study of prospectors, analyzers, and defenders. Information Systems Research.

[7] Niederman, F., Brancheau, J. C., \& Wetherbe, J. C. (1991). Information systems management issues for the 1990s. MIS quarterly.

[8] Porter, M. E. (2002). What is strategy?,

[9] Schmidt, E., \& Cohen, J. (2013). The new digital age: Reshaping the future of people, nations and business. Hachette UK.

[10] Pinto, F. C., Chainho, P., Péssaro, N., Santiago, F., Corujo, D., \& Gomes, D. (2013). The business of things architecture. Transactions on Emerging Telecommunications Technologies, 24(4).

[11] Penny Stocks Lab (2014). Infographic: The Golden Age of Mobile. (Visited in August 2015).

[12] Tansley, A. G. (1935). The use and abuse of vegetational concepts and terms. Ecology, 16(3).

[13] Bosch, J. (2012). Software ecosystems: Taking software development beyond the boundaries of the organization. Journal of Systems and Software, 85.

[14] Messerschmitt, D. G., \& Szyperski, C. (2005). Software ecosystem: Understanding an indispensable technology and industry.

[15] Hax, A. C., \& Wilde II, D. L. (2003). The delta model - A new framework of strategy. Journal of Strategic Management Education.

[16] Wigmore, I. (2013). What is mobile app?.

[17] Fontao, A. L., Santos, R. P., \& Dias-Neto, A. C. (2015). Mobile software ecosystem (MSECO): A systematic mapping study.

[18] Hyrynsalmi, S., Seppänen, M., \& Suominen, A. (2014). Sources of value in application ecosystems. Journal of Systems and Software.

[19] Lim, S. L., \& Bentley, P. J. (2012). App epidemics: Modelling the effects of publicity in a mobile app 
ecosystem. In Artificial Life 13.

[20] Lim, S. L., \& Bentley, P. J. (2013). Investigating app store ranking algorithms using a simulation of mobile app ecosystems. IEEE Congress on Evolutionary Computation.

[21] DBLP. Computer Science Bibliography.

[22] ScienceDirect. Science, Health and Medical Journals, Articles and Books.

[23] CAPES. Journals CAPES/MEC Portal

[24] Google. Google scholar.

[25] Mendeley. Reference Manager and PDF Organizer.

[26] Knight, R. Mendeley desktop 1.10 - progress update.

[27] Petersen, K., Vakkalanka, S., \& Kuzniarz, L. (2015). Guidelines for conducting systematic mapping studies in software engineering: An update. Information and Software Technology.

[28] Sepúlveda, S., Cravero, A., \& Cachero, C. (2016). Requirements modeling languages for software product lines: A systematic literature review. Information and Software Technology.

[29] Office Support. Choosing the best trend line for your data.

[30] Lim, S. L., Bentley, P. J., Kanakam, N., Ishikawa, F., \& Honiden, S. (2015). Investigating Country Differences in Mobile App User Behavior and Challenges for Software Engineering. Software Engineering, IEEE Transactions on, 41(1).

[31] Lim, S. L., Bentley, P. J., \& Ishikawa, F. (2016). The effects of developer dynamics on fitness in an evolutionary ecosystem model of the app store. IEEE Transactions on Evolutionary Computation, 20(4).

[32] Barbosa, O., \& Alves, A. (2011). A systematic mapping study on software ecosystems. Proceedings of the Workshop on Software Ecosystems

[33] Manikas, K., \& Hansen, K. M. (2013). Software Ecosystems - A Systematic Literature Review. DIKU Technical Report.

[34] Guizzardi, G. (2005). Ontological foundations for structural conceptual models. CTIT, Centre for Telematics and Information Technology.

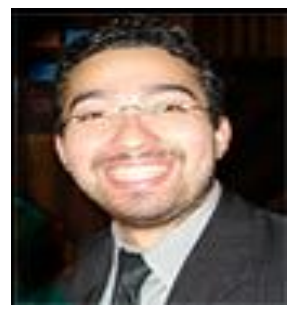

Antoanne Pontes is an MSc student at the Federal University of Rio de Janeiro (UFRJ), Brazil. He holds a postgraduate study in analysis, project and management of information systems from Pontifical Catholic University of Rio de Janeiro (PUC-Rio). His research interests include Mobile App, the adoption of technology and IT strategy.

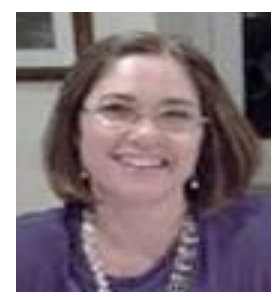

Mônica Ferreira da Silva is a researcher at the Federal University of Rio de Janeiro (UFRJ), Brazil. She received her B.Sc. in mathematics and M.Sc. in system engineering and computer science from UFRJ. She holds a Ph.D. in business administration from the Business School of UFRJ. Her areas of interest include Technology Adoption and IT strategy.

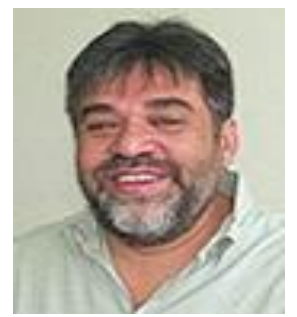

Antonio Juarez Alencar is a researcher at the Federal University of Rio de Janeiro (UFRJ), Brazil. He received his B.Sc. in mathematics and a M.Sc. in system engineering and computer science from UFRJ. He holds a D.Phil. in computer science from Oxford University, England. His research interests include economics of software engineering, IT strategy and risk analysis. 
Eber Assis Schmitz is a professor of computer science at the Federal University of Rio de Janeiro (UFRJ). He holds a B.Sc. in electrical engineering from the Federal University of Rio Grande do Sul (UFRGS), an M.Sc. in electrical engineering from the Federal University of Rio de Janeiro and a Ph.D. in computer science and control from the Imperial College of Science, Technology and Medicine, London, England. His research interests include software development modeling tools, business process modeling and stochastic modeling. 